\title{
Determination of fixed-potential selection using chronoamperometry analysis by screen- printed carbon electrode for rice tungro bacilliform virus (RTBV) detection
}

\begin{abstract}
This paper describes the analysis for the determination of fixed-potential selection using chronoamperometry technique for the detection of rice tungro bacilliform virus (RTBV). RTBV is one of the viruses that cause Tungro disease in rice plantation where the epidemic has been reported since the mid-1960s. Thus immuno-biosensor developed here can replace traditional and conventional analytical systems. One of the important steps to develop immunosensor is to determine the best potential to use in this system to indicate high percentage of active area in conducting electron and it can be used as the best potential for future immunosensor development.
\end{abstract}

Keyword: Rice tungro bacilliform virus (RTBV); Chronoamperometry; Fixed-potential selection; Immunosensor 